\title{
Atividade larvicida do óleo essencial de Rosmarinus officinalis e seu composto majoritário contra Drosophila suzukii
}

\author{
Larvicidal activity of essential oils of Rosmarinus officinalis \\ and their major compound against Drosophila suzukii
}

Michele Trombin de Souza ${ }^{*}$, Mireli Trombin de Souza', Maria Aparecida Cassilha Zawadneak

'Departamento de Patologia Básica, Universidade Federal do Paraná (UFPR), Caixa Postal 19031 CEP 81531-980, Curitiba, PR, Brasil

*autor correspondente $\checkmark$ mictrombin@gmail.com
RESUMO: Drosófila-da-asa-manchada (Drosophila suzukii) é uma praga polífaga que ataca preferencialmente frutos de epiderme fina. Métodos de controle são demandados, uma vez que são escassos os produtos fitossanitários recomendados para essa praga no Brasil. Assim, o objetivo deste trabalho foi avaliar o efeito larvicida do óleo essencial (OE) de Rosmarinus officinalis (alecrim) e de seu constituinte majoritário contra larvas de $D$. suzukii, bem como determinar as alterações morfológicas em órgãos/tecidos. Grupos de 20 larvas foram introduzidos em tubos contendo papel filtro impregnado com OE de $R$. officinalis e eucaliptol $\left(80 \mathrm{mg} \mathrm{L}^{-1}\right)$. Esses produtos ocasionaram mortalidade larval $( \pm 98,5 \%)$ e pupal $( \pm 1,5 \%)$ e inibiram a taxa de pupação $(100 \%)$. Além disso, no cérebro e na cutícula das larvas foram observadas degeneração vacuolar e alterações morfológicas a partir do uso de ambos os produtos. Este trabalho demonstra o uso de OE de $R$. officinalis e eucaliptol como bioinseticidas contra $D$. suzukii, representando uma alternativa aos inseticidas sintéticos para o controle dessa praga.

PALAVRAS-CHAVE: Alecrim, drosófila-daasa-manchada, eucaliptol.

\begin{abstract}
Drosophila suzukii known as spotted-wing-drosophila is a polyphagous pest that preferentially attacks thin-skinned fruits. Control methods are required, since the phytosanitary products recommended for this pest in Brazil are scarce. Thus, the objective of this work was to evaluate the larvicidal effect of the essential oil (EO) of Rosmarinus officinalis (rosemary), and its major compound against D. suzukii larvae, as well as to determine the morphological alterations in organs/tissues. Groups of 20 larvae were introduced into tubes containing filter paper impregnated with R. officinalis EO and eucalyptol (80 $\left.\mathrm{mg} \mathrm{L}^{-1}\right)$. These products caused larval $( \pm 98.5 \%)$ and pupal $( \pm 1.5 \%)$ mortality and inhibited the pupation rate (100\%). In addition, in the brain and cuticle of larvae were observed through vacuolar degeneration and morphological changes with both products. This work demonstrates the use of EO of $R$. officinalis and eucalyptol as bioinsecticides against $D$. suzukii, representing an alternative to synthetic insecticides to control this pest.
\end{abstract}

KEYWORDS: Rosemary, spotted-wing-drosophila, eucalyptol.

\section{Introdução}

A drosófila-da-asa-manchada Drosophila suzukii (Diptera: Drosophilidae) é uma das principais pragas da fruticultura por atacar frutos de epiderme fina, tais como amoras (Morus nigra L.), cerejas (Prunus avium L.), mirtilos (Vaccinium spp.) e morangos (Fragaria $\times$ ananassa Duchesne). Diferindo de outros drosofilídeos, as fêmeas de D. suzukii possuem 
ovipositor duplamente serrilhado capaz de colocar ovos em frutas maduras saudáveis, sendo que ao se alimentarem, suas larvas causam destruição direta dos tecidos. O manejo de D. suzukii é desafiador devido à ampla gama de hospedeiros ( 60 espécies) (ANDREAZZA et al., 2017), ciclo biológico curto (SPITALER et al., 2020) e fácil adaptação ambiental (SÁNCHEZ-RAMOS et al., 2019; SANTOIEMMA et al., 2019; SOUZA et al., 2020a; SPITALER et al., 2020).

Os impactos econômicos dessa praga foram documentados na América do Norte (GOODHUE et al., 2011; FARNSWORTH et al., 2017), América do Sul (SANTOS, 2014) e Europa (DE ROS et al., 2015). No Brasil, as perdas na safra de morango chegaram a até $30 \%$ ao ano (SANTOS, 2014). Na Califórnia, os produtores de framboesa relataram perdas de US $\$ 36,4$ milhões entre 2009 e 2014 (FARNSWORTH et al., 2017), sem contabilizar os custos adicionais gerados pelo monitoramento e controle realizados para prevenir infestações iniciais (GOODHUE et al., 2011).

Apesar dos avanços nas medidas de manejo integrado de D. suzukii (ANDREAZZA et al., 2017), o uso profilático de organofosforados, piretroides e espinosinas sintéticas ainda é a ferramenta mais adotada (GRESS; ZALOM, 2018; SANTOIEMMA et al., 2019; MERMER et al., 2020). Para mitigar esses efeitos, os óleos essenciais (OEs) podem ser uma alternativa ambientalmente segura frente aos sintéticos, pois possuem múltiplos modos de ação, como inibidores da acetilcolinesterase e do ácido gama-aminobutírico e ligantes dos receptores de octopamina (JANKOWSKA et al., 2017). Isso pode diminuir ou evitar a evolução da resistência, assim como uma alta volatilidade que reduz a presença de resíduos nos frutos (SOUZA et al., 2020b, 2021).

Dentre as plantas com potencial de prospecção de moléculas inseticidas destacam-se Rosmarinus officinalis L. (Lamiaceae), popularmente conhecido como alecrim. Estudos fitoquímicos reportaram que o OE de $R$. officinalis tem propriedades contra artrópodes, tais como ação antialimentação, fumigante, larvicidas e repelentes (ISMAN; GRIENEISEN, 2014; TAK; ISMAN, 2015). Em adição, alguns compostos individuais do OE, como o eucaliptol, possuem capacidade de atuar na dissociação dos lipídios presentes na cutícula do exoesqueleto de insetos, provocando a desidratação e a morte da D. suzukii (SOUZA et al., 2020b).

Nesse contexto, o objetivo deste trabalho foi avaliar o efeito larvicida do OE de $R$. officinalis e de seu principal majoritário contra larvas de $D$. suzukii, bem como determinar as alterações morfológicas em órgãos/tecidos.

\section{Metodologia}

\section{Criação de insetos para obtenção do óleo essencial}

Frutos maduros de morangos (Fragaria $\times$ ananassa) da cultivar San Andreas infestados com Drosophila suzukii foram coletados manualmente, entre janeiro e maio de 2018,

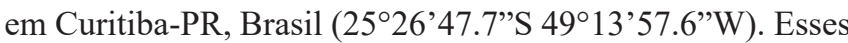
frutos foram colocados em bandejas plásticas $(28,9 \mathrm{~cm}$ de largura $\times 34,9 \mathrm{~cm}$ de comprimento $\times 7,5 \mathrm{~cm}$ de altura) sobre uma camada de vermiculita ( $2 \mathrm{~cm}$ de altura), as quais foram envoltas por sacos de tecido voile para evitar a fuga dos adultos e contaminação com outros insetos (ANDREAZZA et al., 2016). Posteriormente, D. suzukii foi criada em garrafas $(290 \mathrm{~mL})$ $(\mathrm{n}=100$ adultos/por garrafa) contendo dieta artificial à base de farinha de milho, levedura e açúcar (SCHLESENER et al., 2018) e tamponada com algodão hidrofílico. A criação foi mantida em sala climatizada sob $25 \pm 1{ }^{\circ} \mathrm{C}, 70 \pm 5 \%$ de umidade relativa do ar e fotofase de 12 horas para a reprodução dos insetos.

\section{Material vegetal}

As folhas apicais $(2 \mathrm{~kg})$ foram coletadas manualmente a partir de 10 exemplares de Rosmarinus officinalis durante o estágio vegetativo em dezembro de 2014, no período vespertino, às $17 \mathrm{~h}$. As coletas foram realizadas na Área Experimental de Plantas Aromáticas e Medicinais da Fazenda Experimental Canguiri, Universidade Federal do Paraná, Pinhais-PR, Brasil

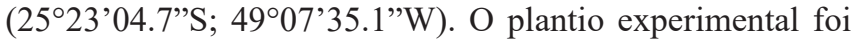
estabelecido em 2011 a partir de transplante das mudas em campo, com o espaçamento de $0,5 \mathrm{~m}$ entre linhas por $0,4 \mathrm{~m}$ entre plantas.

Durante a coleta a temperatura média mensal foi $18,8^{\circ} \mathrm{C}$, a precipitação média mensal foi $115,3 \mathrm{~mm}$ e a umidade relativa de média mensal foi $85,0 \%$ (SISTEMA DE TECNOLOGIA E MONITORAMENTO AMBIENTAL DO PARANÁ, 2014). O solo do cultivo das plantas é classificado como Cambissolo Háplico, com textura argilosa e fertilidade média a alta. Para determinação dos atributos do solo, amostras foram coletadas nas camadas de 0-10 e 10-20 cm de profundidades e encaminhadas para análises físico-químicas (Tabela 1).

\section{Obtenção do óleo essencial}

Para extrair os OEs de $R$. officinalis, as folhas frescas foram homogeneizadas e submetidas a hidrodestilação em aparelho do tipo Clevenger ${ }^{\circledR}$ (AGÊNCIA NACIONAL DE VIGILÂNCIA SANITÁRIA, 2010). Em cada extração dos óleos, $100 \mathrm{~g}$ das folhas, em triplicata, foram colocados em um balão de $2 \mathrm{~L}$

Tabela 1. Características químicas do solo das camadas 0-10 cm e 10-20 cm de profundidade, Pinhais-PR, Brasil, 2014.

\begin{tabular}{|c|c|c|c|c|c|c|c|c|c|c|c|}
\hline \multirow{2}{*}{ cm } & \multirow{2}{*}{$\begin{array}{c}\mathrm{pH} \\
\mathrm{CaCl}_{2}\end{array}$} & \multirow{2}{*}{$\begin{array}{c}\mathrm{P} \\
\mathrm{mg} \mathrm{dm^{-3 }}\end{array}$} & $\mathbf{A l}^{+3}$ & $\mathbf{H}^{+} \mathbf{A l}^{+3}$ & $\mathbf{C a}^{+2}$ & $\mathbf{M g}^{+2}$ & $\mathbf{K}^{+}$ & SB & $\mathbf{T}$ & $\mathbf{V}$ & MO \\
\hline & & & \multicolumn{7}{|c|}{....................cmolc $\mathrm{dm}^{-3} \ldots \ldots \ldots \ldots \ldots \ldots \ldots \ldots$} & \multicolumn{2}{|c|}{$\ldots . . . \% \ldots .}$. \\
\hline 0-10 & 5,0 & 158,4 & $\mathbf{0}$ & 4 & 9,6 & 3,4 & 1,3 & 14,3 & 18,3 & 78 & 6,8 \\
\hline $10-20$ & 5,9 & 168,0 & 0 & 4 & 9,6 & 3,3 & 1,2 & 14,1 & 18,1 & 78 & 5,7 \\
\hline
\end{tabular}

$\mathrm{pH}=$ potencial hidrogeniônico; $\mathrm{P}=$ fósforo extraído por Melich; $\mathrm{Al}^{+3}=$ alumínio; $\mathrm{H}^{+} \mathrm{Al}^{+3}=$ acidez potencial; $\mathrm{Ca}^{+2}=$ cálcio; $\mathrm{Mg}^{+2}=$ magnésio; $\mathrm{K}^{+}=$potássio; $\mathrm{SB}=$ somas de bases; $\mathrm{T}$ = capacidade de troca catiônica efetiva; $\mathrm{V}=$ saturação; $\mathrm{MO}$ = matéria orgânica. 
contendo $1 \mathrm{~L}$ de água destilada. O balão foi aquecido em manta aquecedora, e após iniciar a fervura do material vegetal, este permaneceu por 4 horas para obtenção do $\mathrm{OE}$. O teor foi corrigido para base seca após obter a massa constante de subamostras (20 g), em triplicata, do material vegetal em estufas de ar forçado a $65^{\circ} \mathrm{C}$, até massa constante ( 7 dias). Com base na porcentagem da matéria seca foi calculado o rendimento do óleo essencial (\%) usando a seguinte fórmula:

Rendimento do óleo essencial $(\%)=\frac{\text { massa do óleo essencial obtido }(g)}{\text { massa seca da matéria vegetal }(g)} \times 100$

\section{Análise química dos óleos essenciais}

A análise química do $\mathrm{OE}$ foi realizada conforme protocolo de Trombin-Souza et al. (2017). Para isso, uma alíquota de $1 \mu \mathrm{L}$ das amostras dos OEs foi previamente diluída para uma solução a $1 \%$ em diclorometano com a proporção de divisão de 1:20, seguida de análises por cromatografia em fase gasosa com detecção por ionização em chama (CG-DIC - Agilent 6890) e/ou por espectrometria de massas (CG-EM - Agilent $5973 \mathrm{~N})$ no modo splittless.

Em ambas as análises foi utilizada uma coluna cromatográfica HP-5MS (30 $\mathrm{m} \times 0,25 \mathrm{~mm} \times 0,25 \mu \mathrm{m}$ de espessura de filme) revestida com $5 \%$ fenil-95\% dimetil polissiloxano como fase estacionária). Hélio foi usado como gás de arraste, a uma taxa de fluxo de $1,0 \mathrm{~mL} / \mathrm{min}$. A temperatura inicial do método para as análises cromatográficas foi $50^{\circ} \mathrm{C}$, mantida durante $1 \mathrm{~min}$, seguida de aquecimento a uma taxa de $7{ }^{\circ} \mathrm{C} \min ^{-1}$ até $250{ }^{\circ} \mathrm{C}$, mantida por $10 \mathrm{~min}$. A temperatura do injetor e detector foi mantida a $250^{\circ} \mathrm{C}$, com pressão de $200 \mathrm{kPa}$ por um min no injetor.

A quantificação e identificação dos constituintes químicos dos OEs foram realizadas comparando os seus espectros de massa àqueles de bibliotecas comerciais (MCLAFFERTY; STAUFFER, 2014), bem como por seus índices de retenção linear (VAN DEN DOOL; KRATZ, 1963) após a injeção de uma série de alcanos $\left(\mathrm{C}_{8}-\mathrm{C}_{26}\right)$ e comparados aos dados da literatura (ADAMS, 2007). Todas as amostras foram injetadas em triplicatas.

O constituinte majoritário eucaliptol foi selecionado para os bioensaios realizados, sendo o padrão desta substância (CAS: 470-82-6) obtido da Sigma-Aldrich Brasil com uma pureza de $\geq 99 \%$.

\section{Toxicidade dos óleos essenciais sobre larvas de Drosophila suzukii}

Para os bioensaios de aplicação de contato, grupos de 20 larvas de $D$. suzukii no terceiro instar larval (L3) foram introduzidos em tubos de ensaios de vidro $(5 \mathrm{~mL})$ contendo um papel de filtro ( $2 \mathrm{~cm}$ de largura $\times 8 \mathrm{~cm}$ de comprimento) impregnado com $0,16 \mathrm{~mL}$ do $\mathrm{OE}$ de $R$. officinalis e eucaliptol, solubilizado em $1,84 \mathrm{~mL}$ de acetona pura $(99,5 \%)$. Acetona foi utilizada como controle negativo. Testes anteriores demostraram que esse solvente não interfere na sobrevivência das larvas. Todos os bioensaios foram realizados em condições de laboratório a $25 \pm 2{ }^{\circ} \mathrm{C}, 70 \pm 10 \%$ de umidade relativa e fotofase de 12 horas.

A toxicidade dos OEs sobre $D$. suzukii baseou-se na determinação da concentração letal $\left(\mathrm{CL}_{50} \mathrm{e} \mathrm{CL}_{90}\right)$, que representa uma concentração ou dose capaz de causar mortalidade de $50 \%$ e $90 \%$ da população experimental, respectivamente. Para isso, em cada tratamento foram utilizadas as diluições seriadas dos OEs em acetona pura (99,5\%): 2,5, 5,0, 7,5, 10, 20, $40 \mathrm{e}$ $80 \mathrm{mg} \mathrm{L}^{-1}$. Para a determinação do tempo letal médio $\left(\mathrm{TL}_{50}\right)$ necessário para matar $50 \%$ das larvas de D. suzukii tratadas foi utilizada a concentração máxima de $80 \mathrm{mg} \mathrm{L}^{-1}$ dos OEs de R. officinalis e eucaliptol.

A mortalidade larval (ML), taxa de pupação (TP), mortalidade pupal (MP) foram avaliadas a cada 24 horas durante cinco dias consecutivos, e calculadas conforme Kumar et al. (2014) e Singh e Kaur (2016).

$M L=($ total larvas mortas $\times 100) /$ total de larva testadas

$T P=($ total de pupa $\times 100) /$ total de larva testadas

$M P=($ número de pupas mortas / número de pupas introduzidas $) \times 100$

$\mathrm{O}$ delineamento foi inteiramente casualizado com quatro repetições por tratamentos (OE de R. officinalis e eucaliptol), sendo cada repetição constituída por 20 larvas. Modelos lineares generalizados foram utilizados para a análise das variáveis estudadas, assumindo uma distribuição de Poisson. As médias foram comparadas pelo teste de Tukey $(\mathrm{P}<0,05)$. As análises foram realizadas usando o software estatístico " $R$ " versão 2.15.1 (R DEVELOPMENT CORE TEAM, 2020).

\section{Histologia larval de Drosophila suzukii}

A análises histológicas seguiram o protocolo descrito por Souza et al. (2021). Para isso, duas horas após o contato com OE de $R$. officinalis e eucaliptol, as larvas de D. suzukii $(\mathrm{n}=20)$ foram fixadas em $10 \%$ de formalina tamponada para evitar a autólise dos tecidos. Cinco seções longitudinais foram incluídas em parafina, e as larvas foram seccionadas em série (4 $\mu \mathrm{m}$ de espessura) e coradas com hematoxilina-eosina. Os danos microscópicos em $D$. suzukii foram analisados com auxílio de um microscópio estereoscópico (40× de ampliação) e realizadas as análises descritivas (SOUZA et al., 2021).

\section{Resultados e Discussão}

$\mathrm{O}$ rendimento dos OEs das folhas frescas de $R$. officinalis foi de 1,4\% (Tabela 2). Esse valor foi superior a outros estudos realizados na espécie, que obtiveram entre $0,8 \%$ (MAY et al., 2010) e 1,2\% (AMIN; NAIK; HUSSAIN, 2017). Mediante as análises químicas os picos cromatográficos foram classificados em monoterpenos hidrocarbonetos $(0,8-9,3 \%)$, monoterpenos oxigenados $(0,8-49,1 \%)$, sesquiterpenos hidrocarbonetos $(0,5-0,8 \%)$ e éster $(0,7 \%)$ (Tabela 1$)$. No total, 15 picos cromatográficos foram identificados, entre eles eucaliptol foi o constituinte principal $(49,1 \%)$, representando $98,7 \%$ do total de picos (Figura 1). Como esperado, o OE analisado possui todos os compostos químicos publicados na literatura para a espécie, em que o monoterpeno oxigenado eucaliptol é relatado 
como sendo o constituinte majoritário (ISMAN et al., 2008; BERETTA et al., 2011; KIRAN; PRAKASH, 2015). Entretanto, outros trabalhos identificaram a presença frequente de $\alpha$-pineno, eucaliptol, borneol, acetato de bornila e cânfora como constituintes frequentes (ISMAN et al., 2008; SAYORWAN et al., 2013), corroborando o nosso estudo.

$\mathrm{O}$ perfil químico e as atividades inseticidas do OE de R. officinalis, que têm eucaliptol como constituinte majoritário, foram documentados (ISMAN; GRIENEISEN, 2014; ANDRADE et al., 2018), juntamente com suas propriedades inseticida (ISMAN et al., 2008; KIRAN; PRAKASH, 2015), fumigante (PAPACHRISTOS; STAMPOULOS, 2004; KIRAN; PRAKASH, 2015), repelente (ISMAN et al., 2008), larvicida (TAK; ISMAN, 2015, 2017) e acaricida (CHOI et al., 2004; MIRESMAILLI; ISMAN, 2006). Neste estudo, o OE de R. officinalis e o eucaliptol apresentaram concentração letal (CL) para matar $50 \%$ e $90 \%$ da população testada de 11,03 a $12,10 \mathrm{mg} \mathrm{L}^{-1}$ para $\mathrm{CL}_{50}$, respectivamente, e de 19,48 a $22,24 \mathrm{mg} \mathrm{L}^{-1}$ para $\mathrm{CL}_{90}$, respectivamente (Tabela 3 ). Os resultados também mostraram que o tempo letal $\left(\mathrm{TL}_{50}\right)$ para matar $50 \%$ da população testada foi de 0,55 e 8,10 horas para OE de $R$. officinalis e eucaliptol, respectivamente (Tabela 3). É provável que o menor tempo do OE de R. officinalis para ocasionar a mortalidade das larvas de D. suzukii seja devido ao aumento de penetração dos produtos através da cutícula do inseto como um mecanismo de sinergia entre o eucaliptol e os outros constituintes químicos. Esse efeito sinérgico foi relatado quando o eucaliptol foi usado em mistura com aromadendreno (MULYANINGSIH et al., 2010), limoneno

Tabela 2. Teor de óleo essencial, constituintes identificados, monoterpenos hidrocarbonetos, monoterpenos oxigenados, sesqueiterpenos hidrocarbonetos e éster observados em Rosmarinus officinalis (Lamiaceae).

\begin{tabular}{cc}
\hline Teor de óleo essencial (\%) & 1,4 \\
\hline Total de constituintes identificados (\%) & 98,7 \\
Monoterpenos hidrocarbonetos (\%) & $0,8-9,3$ \\
Monoterpenos oxigenados (\%) & $0,8-49,1$ \\
Sesqueiterpenos hidrocarbonetos (\%) & $0,5-0,8$ \\
Éster (\%) & 0,7 \\
\hline
\end{tabular}

(VUUREN; VILJOEN, 2007) ou outros constituintes menores dos OEs (VILELA et al., 2009; MULYANINGSIH et al., 2010).

O OE de $R$. officinalis e o eucaliptol também proporcionam efeitos nos parâmetros biológicos contra larvas de $D$. suzukii. Observam-se maiores ML e MP, menores TP quando comparados com a acetona (Tabela 4). Essas informações indicam que a ação larvicida desses produtos pode estar relacionada à polaridade dos OEs (substâncias lipofílicas). Fato que permite os óleos penetrarem na cutícula das larvas, interferindo em suas funções fisiológicas e no desenvolvimento dos insetos (KUMAR et al., 2014; SOUZA et al., 2021).

Os danos histológicos foram verificados nas larvas de D. suzukii quando expostas ao OE de $R$. officinalis e eucaliptol, mas não para o grupo de controle (Figura 2 IA e IIA). As alterações incluíram descamação da cutícula (Figura 2 IB) e desprendimento da célula epidermal da camada cuticular (Figura 2 IC). Notam-se também danos cerebrais nas larvas, como intensa vacuolização na camada cortical (Figura 2 IIB) e alteração da morfologia dos neuropilos (Figura 2 IIC), características de degeneração e necrose após os tratamentos. Essas desordens fisiológicas ocasionadas pelo $\mathrm{OE}$ de $R$. officinalis e eucaliptol em larvas de D. suzukii são típicas do processo

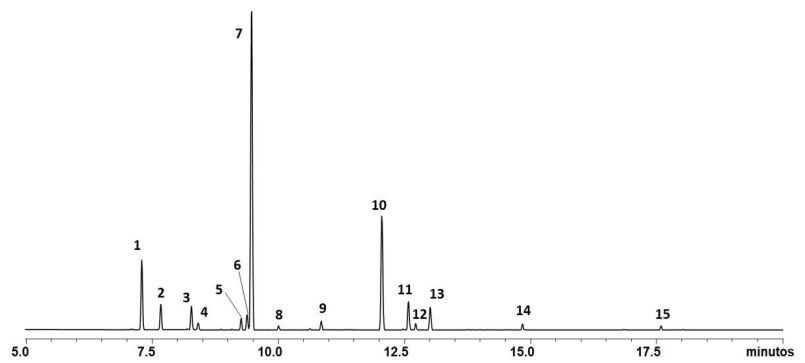

Figura 1. Composição do óleo essencial (\%) das folhas frescas de Rosmarinus officinalis (Lamiaceae) coletadas na região neotropical, Brasil. Monoterpeno hidrocarboneto: 1. $\alpha$-pineno $(9,3 \%)$, 2. canfeno $(3,5 \%), 3$. $\beta$ - $\alpha$-pineno $(3,2 \%), 4$. $\beta$-mirceno $(0,8 \%), 5$. m-cirmeno $(1,5 \%), 6$. limoneno $(2,1 \%)$; Monoterpeno oxigenado: 7. eucaliptol (49,1\%), 8. linalool (1,1\%), 9. borneol $(4,2 \%), 10$. cânfora $(17,8 \%), 11$. terpine-4-ol $(3,3 \%), 12$. $\alpha$-terpineno $(0,8 \%)$; Sesquiterpeno hidrocarboneto: 13 . $\beta$-cariofileno $(0,8 \%), 14$. $\alpha$-cariofileno $(0,5 \%)$; Éster: 15 . acetato de bornila $(0,7 \%)$.

Tabela 3. Toxicidade do óleo essencial de Rosmarinus officinalis (alecrim) e eucaliptol sobre larvas de Drosophila suzukii após 72 horas da exposição inicial.

\begin{tabular}{|c|c|c|c|c|c|}
\hline Produtos & $n^{\mathrm{a}}$ & $\begin{array}{c}\text { Coeficiente } \\
\text { angular } \pm \mathbf{E P}\end{array}$ & $\mathrm{CL}_{\mathbf{5 0}}(\mathbf{9 5 \%} \mathrm{IC})^{B}$ & $\mathrm{CL}_{\mathbf{9 0}}(\mathbf{9 5 \%} \mathrm{IC})^{B}$ & $\mathbf{G} \mathbf{L}^{C}$ \\
\hline \multicolumn{6}{|c|}{ Concentração letal } \\
\hline alecrim & 80 & $4,10 \pm 0,11$ & $11,03(10,62-11,76)$ a & $\begin{array}{c}19,48(19,22 \\
-20,61) a\end{array}$ & 1 \\
\hline eucaliptol & 80 & $3,22 \pm 0,86$ & $12,10(11,94-13,08) \mathrm{b}$ & $\begin{array}{c}22,24(21,89- \\
22,82) \text { b }\end{array}$ & 1 \\
\hline \multicolumn{6}{|c|}{ Tempo letal médio } \\
\hline Tratamentos & $n^{\mathrm{a}}$ & \multicolumn{2}{|c|}{ Coeficiente angular $\pm \mathrm{EP}$} & $\mathrm{TL}_{\mathbf{5 0}}(\mathbf{9 5 \%} \mathrm{IC})^{D}$ & $\mathbf{G L}^{C}$ \\
\hline alecrim & 80 & \multicolumn{2}{|c|}{$4,26 \pm 0,32$} & $0,55(0,46-0,68)$ a & 1 \\
\hline eucaliptol & 80 & \multicolumn{2}{|c|}{$3,67 \pm 0,14$} & $-8,22) b$ & 1 \\
\hline
\end{tabular}

${ }^{A}$ Número de insetos por tratamento. ${ }^{B}$ Concentração letal $\left(\mathrm{mg} \mathrm{L}^{-1}\right)$ necessária para ocasionar $50 \% \mathrm{e} 90 \%$ de mortalidade das larvas; (IC $=$ intervalo de confiança). ${ }^{C} \mathrm{Graus}$ de liberdade. ${ }^{D}$ Tempo letal (horas) necessário para ocasionar $50 \%$ das larvas; $(\mathrm{IC}=$ intervalo de confiança). $\pm \mathrm{EP}: \pm$ erro padrão. $P>0,05$ no teste de comparação de médias. As colunas seguidas pela mesma letra não são significativamente diferentes entre si pelo teste de qui-quadrado 
Tabela 4. Mortalidade larval (ML), taxa de pupação (TP), mortalidade pupal (MP) de Drosophila suzukii tratados com óleo essencial de Rosmarinus officinalis (alecrim) e eucaliptol.

\begin{tabular}{|c|c|c|c|}
\hline \multirow{2}{*}{ Produtos } & ML & $\mathbf{T P}$ & MP \\
\hline & \multicolumn{3}{|c|}{ 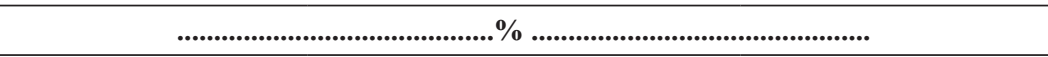 } \\
\hline Alecrim & $98,0 \pm 1,22 a$ & $2,0 \pm 1,22 b$ & $100,0 \pm 0,00 a$ \\
\hline Eucaliptol & $99,0 \pm 1,00 a$ & $1,0 \pm 1,00 \mathrm{~b}$ & $100,0 \pm 0,00 a$ \\
\hline Acetona & $\mathbf{0 , 0 0} \pm \mathbf{0 , 0 0} b$ & $100,0 \pm 0,00 a$ & $\mathbf{0 , 0 0} \pm \mathbf{0 , 0 0} b$ \\
\hline $\mathbf{F}$ & 11,23 & 24,11 & 7,35 \\
\hline GL & 2 & 2 & 2 \\
\hline $\mathbf{P}$ & $<0,0001$ & $<0,0001$ & $<0,0001$ \\
\hline
\end{tabular}

As colunas seguidas pela mesma letra não são significativamente diferentes entre si pelo teste de Tukey: P < 0,05. F: teste-F; GL: Grau de Liberdade; P: probabilidade

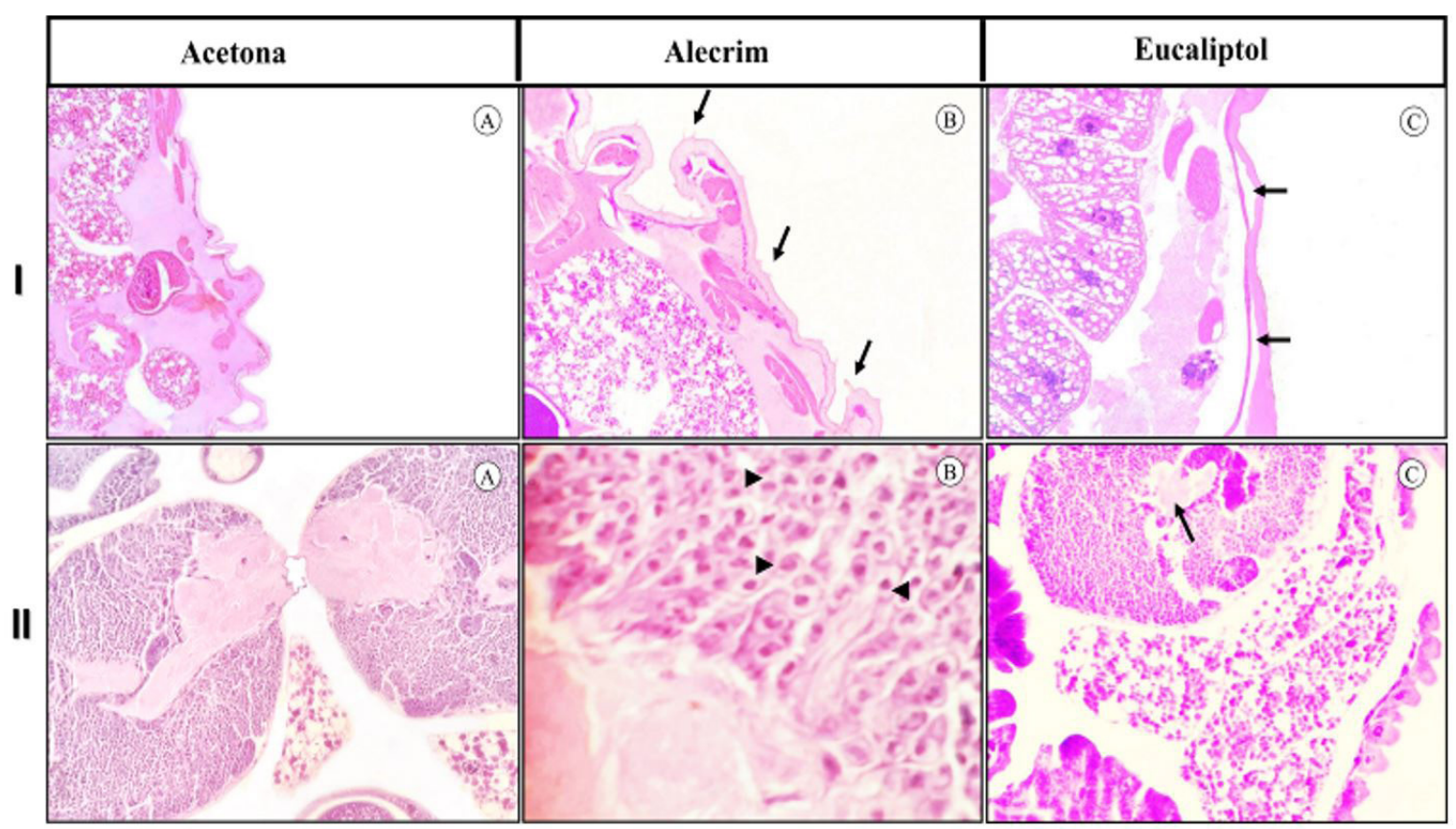

Figura 2. Microscopia de cutícula (I) e cérebro (II) de Drosophila suzukii tratados com acetona (A) e óleo essencial de Rosmarinus officinalis - alecrim (B) e eucaliptol (C).

de morte celular (KERR, 2002). Estudos relacionados a danos histopatológicos causados por OEs concluíram que o modo de ação é semelhante, independentemente da taxonomia do inseto-alvo (CHAABAN et al., 2018; CHAABAN et al., 2019).

Nesse contexto, levando-se em consideração a elevada toxicidade do OE de $R$. officinalis e de seu constituinte majoritário eucaliptol sobre larvas de D. suzukii, seu uso poderá ser alternativa viável, sustentável e com capacidade de gerar produtos naturais compatíveis com a entomofauna benéfica. Assim, este estudo pode contribuir futuramente para o desenvolvimento de novos produtos para o manejo de D. suzukii, principalmente devido ao $R$. officinalis ser abundantemente cultivado na região neotropical.

\section{Conclusão}

O OE de Rosmarinus officinalis e o eucaliptol ocasionam mortalidade larval e pupal e inibem a pupação, além de causarem danos à cutícula e ao cérebro das larvas de D. suzukii.

\section{Agradecimentos}

Os autores agradecem ao Conselho Nacional de Desenvolvimento Científico e Tecnológico (CNPq), à Coordenação de Aperfeiçoamento de Pessoal de Nível Superior (Capes) e à Fundação Araucária pelas bolsas e suportes financeiros para a realização desta pesquisa.

\section{Referências}

Adams, R. P. Identification of essential oil components by Gas Chromatography/Mass Spectorscopy. New York: Texensis Publishing, 2007.

AGÊNCIA NACIONAL DE VIGILÂNCIA SANITÁRIA-ANVISA.

Farmacopéia Brasileira. Brasília: ANVISA, 2010. v. 33, p. 524.

Amin, T.; Naik, H. R.; Hussain, S. Z. Chemotyping the essential oil in different rosemary (Rosmarinus officinalis L.) plants grown in Kashmir Valley. Biosciences Biotechnology Research Asia, India, v. 14, n. 3, p. 1025-1031, 2017. 
Andrade, J. M. et al. Rosmarinus officinalis L.: an update review of its phytochemistry and biological activity. Future Science OA, London, v. 1, n. 4, p. FSO283, 2018.

Andreazza, F. et al. Drosophila suzukii in Southern Neotropical Region: current status and future perspectives. Neotropical Entomology, Londrina, v. 46, p. 591-605, 2017.

Andreazza, F. et al. Técnica de criação de Drosophila suzukii (Matsumura, 1931) (Diptera: Drosophilidae) em dieta artificial. Boletim de Pesquisa e Desenvolvimento, Brasília, v. 240, p. 1-25, 2016.

Beretta, G. et al. An analytical and theoretical approach for the profiling of the antioxidant activity of essential oils: the case of Rosmarinus officinalis L. Journal of Pharmaceutical and Biomedical Analysis, Oxford, v. 55, n. 5, p. 1255-1264, 2011.

Chaaban, A. et al. Essential oil from Curcuma longa leaves: can an overlooked by-product from turmeric industry be efective for myiasis control? Industrial Crops and Products, Netherlands, v. 132, p. 352-364, 2019.

Chaaban, A. et al. Tissue damage and cytotoxic effects of Tagetes minuta essential oil against Lucilia cuprina. Experimental Parasitology, United States, v. 198, p. 46-52, 2018.

Choi, W. I. et al. Toxicity of plant essential oils to Tetranychus urticae (Acari: Tetranychidae) and Phytoseiulus persimilis (Acari: Phytoseiidae). Journal of Economic Entomology, United Kingdom, v. 97, n. 2, p. 553-558, 2004.

De Ros, G. et al. The economic impact of invasive pest Drosophila suzukii on berry production in the Province of Trento, Italy. Journal of Berry Research, Amsterdam, v. 5, n. 2, p. 89-96, 2015.

Farnsworth, D. et al. Economic analysis of revenue losses and control costs associated with the spotted wing drosophila, Drosophila suzukii (Matsumura), in the California raspberry industry. Pest Management Science, United Kingdom, v. 73, n. 6, p. 1083-1090, 2017.

Goodhue, R. E. et al. Spotted wing drosophila infestation of California strawberries and raspberries: economic analysis of potential revenue losses and control costs. Pest Management Science, United Kingdom, v. 67, n. 11, p. 1396-1402, 2011.

Gress, B. E.; Zalom, F. G. Identification and risk assessment of spinosad resistance in a California population of Drosophila suzukii. Pest Management Science, United Kingdom, v. 75, n. 5, p. 1270-1276, 2018.

Isman, M. B.; Grieneisen, M. L. Botanical insecticide research: many publications, limited usefull data. Trends in Plant Science, United States, v. 19, n. 3, p. 140-145, 2014.

Isman, M. B.; Wilson, J. A.; Bradbury, R. Insecticidal activities of commercial rosemary oils (Rosmarinus officinalis) against larvae of Pseudaletia unipuncta. and Trichoplusia ni. in relation to their chemical compositions. Pharmaceutical Biology, United Kingdom, v. 46, n. 1, p. 82-87, 2008.

Jankowska, M. et al. Molecular targets for components of essential oils in the insect nervous system-a review. Molecules, Basel, v. 33, n. 1, p. 34, 2017.

Kerr, J. F. R. History of the events leading to the formulation of the apoptosis concept. Toxicology, Netherlands, v. 181-182, n. 27, p. 471-474, 2002.

Kiran, S.; Prakash, B. Toxicity and biochemical efficacy of chemically characterized Rosmarinus officinalis essencial oil against Sitophilus oryzae and Oryzaephilus surinamensis. Industrial Crops and Products, Netherlands, v. 74, n. 15, p. 817-823, 2015.
Kumar, P. et al. Biocontrol potential of essential oil monoterpenes against housefly, Musca domestica (Diptera: muscidae). Ecotoxicology and Environmental Safety, Orlando, v. 100, p. 1-6, 2014.

May, A. et al. Produção de biomassa e óleo essencial de alecrim (Rosmarinus officinalis L.) em função da altura e intervalo entre cortes. Revista Brasileira de Plantas Medicinais, Botucatu, v. 12, n. 2, p. 195-200, 2010.

Mclafferty, F. W.; Stauffer, D. B. Unknown identification using reference mass spectra. Quality evaluation of databases. Journal of the American Society for Mass Spectrometry, United States, v. 10, p. 1229-1240, 1999.

Mermer, S. et al. Timing and order of different insecticide classes drive control of Drosophila suzukii; a modeling approach. Journal of Pest Science, Heidelberg, v. 94, p. 743-755, 2020.

Miresmailli, S.; Isman, M. B. Efficacy and persistence of rosemary oil as an acaricide against twospotted spider mite (Acari: Tetranychidae) on greenhouse tomato. Journal of Economic Entomology, United Kingdom, v. 99, n. 6, p. 2015-2023, 2006.

Mulyaningsih, S. et al. Synergistic properties of the terpenoids aromadendrene and 1,8-cineole from the essential oil of Eucalyptus globulus against antibiotic-susceptible and antibiotic-resistant pathogens. Phytomedicine, Germany, v. 17, n. 13, p. 1061-1066, 2010.

Papachristos, D. P.; Stampoulos, D. C. Fumigant toxicity of three essential oils on the eggs of Acanthoscelides obtectus (Say) (Coleoptera: Bruchidae). Journal of Stored Products Research, United Kingdom, v. 40, n. 5, p. 517-525, 2004.

R DEVELOPMENT CORE TEAM. A language and environment for statistical computing. Vienna, Austria: R Foundation for Statistical Computing. Disponível em: http://www.R-project.org. Acesso em: 23 de ago. 2020.

Sánchez-Ramos, I. et al. Reproductive potential and population increase of Drosophila suzukii at constant temperatures. Entomologia Generalis, Germany, v. 39, n. 2, p. 103-115, 2019.

Santoiemma, G. et al. Habitat preference of Drosophila suzukii across heterogeneous landscapes. Journal of Pest Science, Germany, v. 92, n. 2, p. 485-494, 2019.

Santos, R. S. S. Ocorrência de Drosophila suzukii (Matsumura, 1931) (Diptera: Drosophilidae) atacando frutos de morango no Brasil. Comunicado Técnico 159, Bento Gonçalves, v. 1, p. 1-4, 2014.

Sayorwan, W. et al. Effects of inhaled rosemary oil on subjective feelings and activities of the nervous system. Scientia Pharmaceutica, Switzerland, v. 81, n. 2, p. 531-542, 2013.

Schlesener, D. C. H. et al. Biology and fertility life table of Drosophila suzukii on artificial diets. Entomologia Experimentalis et Applicata, United Kingdom, v. 116, n. 11-12, p. 932-936, 2018.

Singh, A.; Kaur, J. The bioefficacy of crude extracts of Azadirachta indica (Meliaceae) on the survival and development of myiasiscausing larvae of Chrysomya bezziana (Diptera: Calliphoridae). Tropical Animal Health and Production, Edinburgh, v. 48, n. 1, p. 117-124, 2016.

SISTEMA DE TECNOLOGIA E MONITORAMENTO AMBIENTAL DO PARANÁ - SIMEPAR. Dados de temperatura média, precipitação mensal e umidade relativa, município de Pinhais. Curitiba: SIMEPAR, 2014.

Souza, M. T. et al. Physicochemical characteristics and superficial damage modulate persimmon infestation by Drosophila suzukii and Zaprionus indianus (Diptera: Drosophilidae). Environmental Entomology, Oxford, v. 49, p. 1290-1299, 2020a. 
Souza, M. T. et al. Chemical composition of essential oils of selected species of Piper and their insecticidal activity against Drosophila suzukii and Trichopria anastrephae. Environmental Science and Pollution Research International, Landsberg, v. 27, p. 13056-13065, 2020b.

Souza, M. T. et al. Insecticidal and oviposition deterrent effects of essential oils of Baccharis spp. and histological assessment against Drosophila suzukii (Diptera: Drosophilidae). Scientific Reports, London, v. 11, n. 3944, p. 1-15, 2021.

Spitaler, U. et al. Yeast species affects feeding and fitness of Drosophila suzukii adults. Journal of Pest Science, Heidelberg, v. 93, p. 1295-1309, 2020.

Tak, J. H.; Isman, M. B. Enhanced cuticular penetration as the mechanism for synergy of insecticidal constituents of rosemary essential oil in Trichoplusia ni. Scientific Reports, London, v. 5, p. 12690, 2015.

Tak, J. H.; Isman, M. B. Penetration-enhancement underlies synergy of plant essential oil terpenoids as insecticides in the cabbage looper, Trichoplusia ni. Scientific Reports, London, v. 7, p. 42432, 2017.

Trombin-Souza, M. et al. Chemical composition of the essential oils of Baccharis species from southern Brazil: a comparative study using multivariate statistical analysis. The Journal of Essential Oil Research, United Kingdom, v. 29, p. 400-406, 2017.

Van Den Dool, H.; Kratz, P. D. J. A. A generalization of the retention index system including linear temperature programmed gas-liquid partition chromatography. Journal of Chromatography. A, Amsterdam, v. 11, p. 463-471, 1963.

Vilela, G. R. et al. Activity of essential oil and its major compound, 1,8-cineole, from Eucalyptus globulus Labill., against the storage fungi Aspergillus flavus Link and Aspergillus parasiticus Speare. Journal of Stored Products Research, Amsterdam, v. 45, n. 2, p. 108-111, 2009

Vuuren, S. F.; Viljoen, A. M. Antimicrobial activity of limonene enantiomers and 1,8-cineole alone and in combination. Flavour and Fragrance Journal, Chichester, v. 22, n. 6, p. 540-544, 2007.

Recebido: 05 abr. 202 Aprovado: 05 nov. 2021 